\title{
Basic Cognitive Architecture, Systemic Inflammation, and immune Dystunction in Schizophrenia
}

Dol. $10.17691 / \mathrm{stm} 2019,11.3 .04$

Received February 6, 2019

V.L. Ushakov, PhD, Associate Professor, Leading Researcher ${ }^{1}$; Senior Researcher ${ }^{2}$;

I.K. Malashenkova, MD, PhD, Head of the Laboratory of Molecular Immunology and Virology";

S.A. Krynskiy, MD, PhD, Junior Researcher';

S.I. Kartashov, Research Engineer ${ }^{1,2}$;

V.A. Orlov, Research Engineer ${ }^{1}$;

D.G. Malakhov, Research Engineer ${ }^{1}$;

N.A. Hailov, MD, PhD, Senior Researcher';

D.P. Ogurtsov, MD, PhD, Laboratory Researcher ${ }^{1,3}$;

N.V. Zakharova, MD, PhD, Associate Professor"; Senior Researcher ${ }^{5}$;

N.A. Didkovsky, MD, DSc, Professor, Head of the Laboratory of Clinical Immunology4;

A.V. Maslennikova, PhD, Researcher ${ }^{6}$;

A.Y. Arkhipov, Junior Researcher ${ }^{6}$;

V.B. Strelets, DSc, Chief Researcher ${ }^{6}$;

B.M. Velichkovsky, DSc, Professor, Corresponding Member of the Russian Academy of Sciences,

Chief Researcher ${ }^{1,7}$; Senior Professor ${ }^{8}$;

Yu.I. Kholodny, PhD, DSc, Chief Specialist ${ }^{1}$; Professor ${ }^{9}$;

G.P. Kostyuk, MD, DSc, Professor, Chief Physician ${ }^{5}$

${ }^{1}$ National Research Center "Kurchatov Institute", 1 Akademika Kurchatova Square, Moscow, 123182, Russia;

${ }^{2}$ National Research Nuclear University MEPhl, 31 Kashirskoe Shosse, Moscow, 115409, Russia;

${ }^{3}$ Federal Research and Clinical Center of Physical-Chemical Medicine, Federal Medical Biological Agency of Russia,

1A Malaya Pirogovskaya St., Moscow, 119435, Russia;

${ }^{4}$ Pirogov Russian National Research Medical University, 1 Ostrovitianov St., Moscow, 117997, Russia;

${ }^{5}$ Alekseev Psychiatric Clinical Hospital No.1, Moscow Department of Health, 2 Zagorodnoe Shosse, Moscow, 117152, Russia;

${ }^{6}$ Institute of Higher Nervous Activity and Neurophysiology, Russian Academy of Sciences, 5A Butlerova St., Moscow, 117485, Russia;

${ }^{7}$ Russian State University for the Humanities, 6 Miusskaya Square, Moscow, 125993, Russia;

${ }^{8}$ Technische Universität Dresden, Zellescher Weg 17, Room A221, Dresden, 01069, Germany;

${ }^{9}$ Bauman Moscow State Technical University (National Research University), 5/1 Baumanskaya 2-ya St.,

Moscow, 105005, Russia

The aims of the project were to study the architecture of the brain's neural networks in the process of perceiving individual stimuli that are personally significant, to evaluate the main parameters of immunity and systemic inflammation, and to relate those to brain damage markers and patterns of cognitive-affective disorders in patients with schizophrenia.

Materials and Methods. The study involved 35 patients with paranoid schizophrenia and 17 people without cognitive impairment. Clinical information about patients was obtained from the database created in the Alekseev Psychiatric Clinical Hospital No.1, Moscow Department of Health. The immunological arm of the study included the determination of key cytokines, neurotrophic factors, circulating immune complexes, C-reactive protein, cortisol, and immunoglobulins in the blood serum of patients. To study the cognitive functions, original methods of selecting the stimuli and recording the vegetative reactions were used; those allowed for identifying the architecture of neural networks involved in the pathogenesis of schizophrenia.

Corresponding author: Vadim L. Ushakov, e-mail: ushakov_VL@nrcki.ru 
Results. In this study, for the first time, changes in humoral immunity and parameters of systemic inflammation were found in patients with schizophrenia. Those changes included an increase in the levels of cytokines IL-6, IL-8, and interferon $\gamma$, persisted regardless of the duration and nature of the antipsychotic therapy. Moreover, the immunological deviations were associated with the phase of the disease, it indicating their endogenous nature and connection with the pathogenesis of schizophrenia.

Our pilot neuroimaging studies showed the feasibility of using MRI-compatible polygraphy for creating a group of fMRI images suitable for statistical analysis thus providing an objective assessment of physiological reactions to the presentation of personally-significant stimuli (based on keywords and images from the patient's anamnesis). By using this approach, we were able to detect the activation of neural networks in the associative zones of the left cuneus and precuneus and the ventrolateral prefrontal cortex of both hemispheres, i.e., the areas responsible for the working memory and processes of consciousness.

Conclusion. Important novel data have been obtained on the relations between neurophysiological parameters, the cytokine profile and the immune status in schizophrenia.

Key words: systemic inflammation; humoral immunity; neurotrophic factors; fMRI; neural networks; schizophrenia; cognitive architecture; architecture of neural networks.

\section{Introduction}

Schizophrenia is a polymorphic mental disorder with characteristic impairments in thinking, perception and cognitive abilities: memory, attention, and executive functions. The socio-economic burden of schizophrenia stems from the high percentage of disability in the affected individuals of working age and the high costs of treatment and/or maintenance of patients [1].

Schizophrenia is considered to be a multifactorial disease; its course depends on genetic factors and also on adverse environmental (psychosocial) factors [2]. There is no single universally accepted theory of the pathogenesis of schizophrenia. Neuroimaging methods reveal structural and functional changes in the patient brains: a decrease in the number of neurons and a change in the response to neurotransmitters. Investigations using positron emission tomography revealed an imbalance in the metabolism of various zones of the cortex, as well as an increase in the expression of dopamine in the brains of patients with schizophrenia [3]. Also, some reports suggest that impaired functional connectivity of neural networks plays an important role in producing the symptoms of schizophrenia [4-7].

Currently, neuroinflammation, systemic inflammatory response, and immune disorders are commonly accepted to be part of schizophrenia. Thus, signs of CNS inflammation with chronic hyper-activation of microglia and astrocytes were found in patients with schizophrenia [8]. As suggested, neuroinflammation in schizophrenia can be infectious, autoimmune or traumatic in nature [8, 9]. The role of systemic inflammation in the pathogenesis of this disease has been also discussed [10]. In this regard, it should be noted that most results on the cellular and molecular mechanisms of schizophrenia were obtained from post-mortal brain studies, cell cultures, and animal models. To translate these data to the clinical situation may be difficult because of the heterogeneity of schizophrenia risk factors and the variety of the clinical forms of the disease.

In the recent literature, there are practically no interdisciplinary studies on the immune and neuroimmune systems in association with other pathogenetic factors of schizophrenia. There is a need to study the schizophreniaassociated immune disorders and systemic inflammation. In addition, the time has come to combine the biomedical research with the modern methods of neuroscience (neurophysiological studies, intravital neuroimaging functional MRI (fMRI), etc.). This article presents the progress results of an interdisciplinary project, where, for the first time, patients with schizophrenia were concomitantly tested for their immunity status, systemic inflammation, peripheral neuroproteins, and fMRI parameters with the help of a comprehensive clinical study and neuropsychological testing.

The aims of the project were to study the architecture of the brain's neural networks in the process of perceiving individual stimuli that are personally significant, to evaluate the main parameters of immunity and systemic inflammation, and to relate those to brain damage markers and patterns of cognitive-affective disorders in patients with schizophrenia.

\section{Materials and Methods}

The study included 35 patients with paranoid schizophrenia from 18 to 42 years old (average age $-29 \pm 2$ years) with the onset of prodromal events (initial stage) at $18 \pm 6$ years of age, with the first manifestation - at $24 \pm 6$ years, and the onset of the continuous stage - at $22 \pm 6$ years. At the time of this study, the antipsychotic therapy was maintained for 6 months to 2 years.

The control group included 17 healthy subjects without cognitive or mental impairment (9 men, 16 women) from 23 to 33 years old with an average age of $27 \pm 4$ years. To accumulate more data on the normal immunological background, the results of another 40 healthy subjects were added to our analysis; this group included 21 women and 19 men with an average age of $39 \pm 5$ years, who were previously examined in the laboratory of molecular immunology and virology at the National Research Center "Kurchatov Institute". 
The criteria for inclusion in the study for patients of the main group were the diagnosis of "paranoid schizophrenia", the ability to sign and date the informed consent form, and the age under 42 years. In all cases, voluntary informed consent was obtained after the explanation of the procedure and the study design. The work was approved by the local Ethics Committee of the National Research Center "Kurchatov Institute".

The study was based on the complete clinical information obtained from the patient records held in the Alekseev Psychiatric Clinical Hospital No.1, Moscow Department of Health. The data array included more than 1000 variables for each patient. The clinical data were collected from individuals who applied for psychiatric care and were treated in this hospital. The database contained clinical (psychopathological), sociodemographic, and psychometric data of patients with schizophrenia spectrum disorders.

We measured the serum levels of cytokines (IL-6, IL-8, IL-10), interferon y (IFN-y), tumor necrosis factor $\alpha$ (TNF- $\alpha$ ), IL-1 $\beta$ IL-1RA receptor antagonist, circulating immune complexes, C-reactive protein, cortisol and immunoglobulins (IgA, IgM, IgG) - by using enzymelinked immunosorbent assays (ELISA) (Cytokine and Hema, Russia). To determine the level of neurotrophic factors in the blood serum, ELISA kits manufactured by R\&D Systems (USA) for brain-derived neurotrophic factor (BDNF), RayBiotech (USA) for nerve growth factor $\beta$ (NGF- $\beta$ ) and neurotrophin-4 (NT-4) were used.

Statistical data processing was performed with the standard software packages Excel (Microsoft, 2010) and Statistica 10.0 (StatSoft Inc., 2010) using the standard statistical parameters. To characterize the results, the mean values $(\mathrm{M})$ and standard errors of the mean $(\mathrm{m})$ were used. The differences between two values were considered statistically significant at a significance level (p) of less than 0.05 according to the Student's criterion.

According to the published reports, the structural and functional changes in the brain are sensitive parameters in schizophrenia [11]. To monitor these changes, we proposed an original method for selecting a personally-significant audio/video stimulus related to the background of patient's hallucinatory-delusional syndrome (based on keywords and images from the patient's anamnesis). To determine the vegetative parameters, the method of MRI-compatible polygraphy was adapted [12]. These methods (approaches) were expected to allow for assessing the impact of personallysignificant stimuli on the examined subject. Thus, for statistical comparison, several groups of these stimuli were created. The first group included all personally significant stimuli presented to the subject, and the second group - the stimuli from the first group, which had a personal significance for the subject as confirmed by the objective data from the polygraph.

The stimuli were presented in pairs for both the patients and healthy subjects of the control group. We used the block paradigm of the stimulus presentation.
At each fMRI test, subjects were asked to relax for $20 \mathrm{~s}$ prior to the stimulus presentation. Each fMRI study included three blocks lasting 64 s. Each block included the presentation of personally significant visual or auditory stimuli for $16 \mathrm{~s}$ followed by $16 \mathrm{~s}$ of rest and the presentation of neutral stimuli for $16 \mathrm{~s}$ and $16 \mathrm{~s}$ of rest. The study was conducted using a Magnetom Verio 3T magnetic resonance imager (Siemens, Germany) with a 32-channel head MRI coil. To record the BOLD (blood oxygen level-dependent) signal while presenting the visual stimuli, we used the standard echo-planar sequence (time repetition, TR=2000 ms; voxel size $2 \times 2 \times 2 \mathrm{~mm}$ ). High resolution anatomical data were also obtained for each subject based on the T1-weighted sequence ( $T R=1900 \mathrm{~ms}$; time echo, $T E=2.21 \mathrm{~ms}$; 176 slices; voxel size $-1 \times 1 \times 1 \mathrm{~mm})$.

For each of the block paradigms, pairwise comparisons were made using the Student criterion; then, with the help of the SPM12 program, individual statistical maps were created. For each of the two compared groups - controls and patients, statistical maps were calculated based on the one-sample t-test. Intergroup comparisons were based on the two-sample t-test. The obtained statistical maps were placed on a T1-weighted image; that allowed us to attribute the active voxels to certain anatomical structures in the AAL2 atlas.

\section{Results and Discussion}

Characteristics of immunity, systemic inflammation and the level of major cytokines. Currently, it is believed that psychotropic therapy suppresses the activity of the immune system. In this study, however, despite of clinical improvement during the treatment in the hospital, the majority of patients showed elevated markers of systemic inflammation and activation of humoral immunity. Thus, among the main immunoglobulins (IgA, $\lg M$, and $\lg G)$ in patients with paranoid schizophrenia, the level of total $\lg M$ in the blood serum increased (Figure 1); the levels of $\lg A$ and $\lg G$ also increased in comparison with the normal values, but not versus those in the control group. An increased content of $\operatorname{lgG}$ - the main protective immunoglobulin of adaptive immunity - was observed in 21 of 35 patients with schizophrenia.

In patients with paranoid schizophrenia, an increase in the main markers of the systemic inflammatory response was found: the level of C-reactive protein, cortisol, as well as the pro-inflammatory cytokines IL-6, IL-8, and IFN-y significantly increased compared with both the control group and the normal values (Figures 2, 3). The level of IL-8 increased in 27 of 35 cases, IL- 6 in 26 of 35 cases, and IFN-y in 19 of 35 cases $(p<0.05)$. The increase in the concentration of cortisol (the stress and anti-inflammatory hormone) was also significant (see Figure 2).

The changes in the inflammation and humoral immunity markers in patients with schizophrenia 


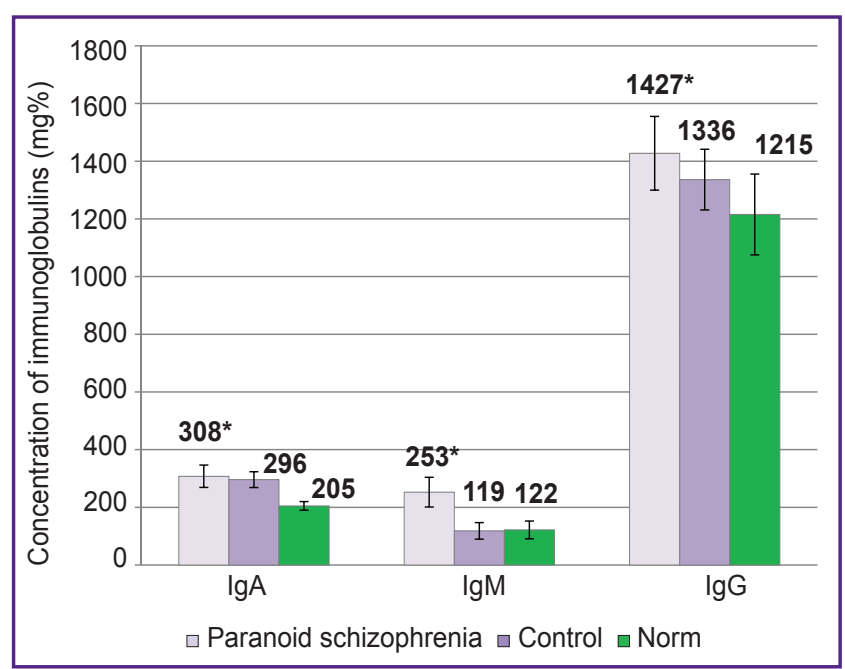

Figure 1. Concentrations of immunoglobulins $A, M, G$ in patients with paranoid schizophrenia $(n=35)$, in the control group $(n=17)$ and in healthy subjects $(n=40) ;{ }^{*}$ statistically significant differences from the norm $(p<0.05)$

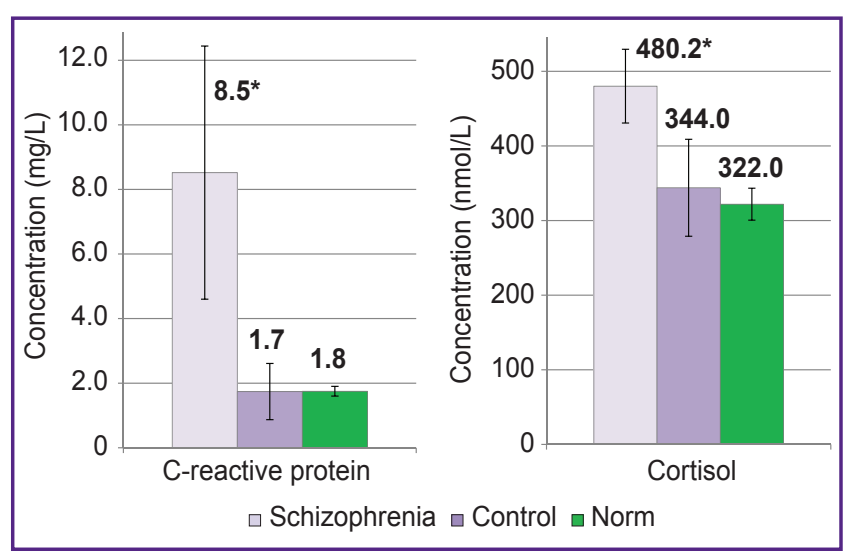

Figure 2. Concentrations of $\mathrm{C}$-reactive protein and cortisol in patients with schizophrenia $(n=35)$, in the control group $(n=17)$ and in healthy subjects $(n=40) ;{ }^{*}$ statistically significant differences from the norm $(p<0.05)$

indicate that these patients experienced active systemic inflammation, despite the antipsychotic therapy and positive clinical effect.

On average, the levels of neurotrophic factors: NT-4, NGF- $\beta$, and BDNF in the blood of patients with schizophrenia did not differ from the normal ones, apparently due to high individual variations of the measured values. Thus, the level of NT-4 was below normal in 18 people, in 8 it was increased, and only in 9 people it was within the normal limits. An increase in NGF- $\beta$ above $15 \mathrm{pg} / \mathrm{ml}$ was detected in $25 \%$ of cases (9 people); in other cases it was normal. The level of BDNF showed less variability: it was increased in 6 patients, decreased in 7 of them, and on average for the group, it did not differ from the norm. A preliminary analysis found that the levels of NT-4 and

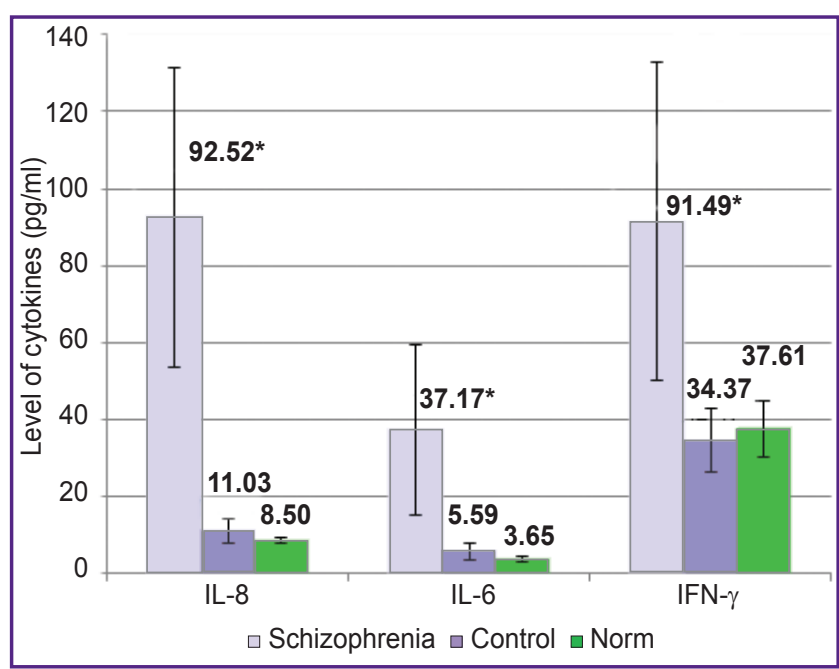

Figure 3. Levels of cytokines IL-8, IL-6, IFN-y in schizophrenia $(n=35)$ compared with the control group $(n=17)$ and normal values $(n=40) ;{ }^{*}$ statistically significant differences from the norm $(p<0.05)$

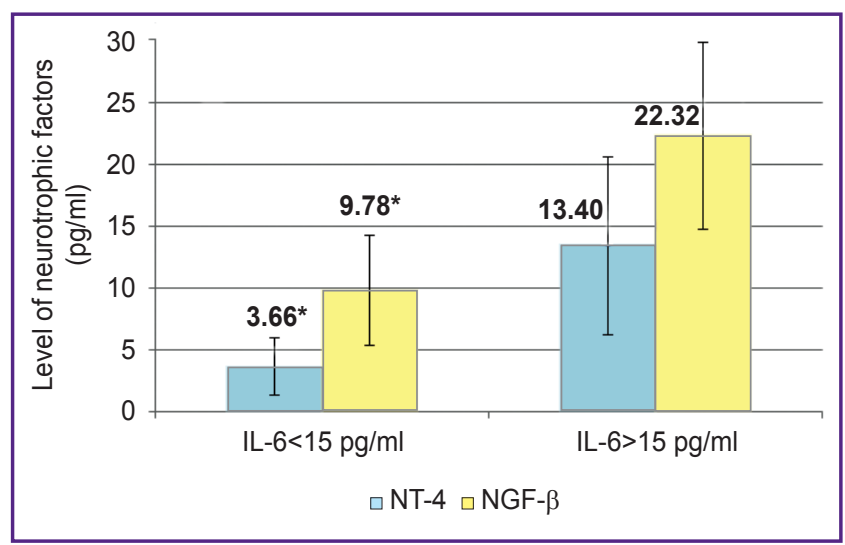

Figure 4. Levels of neurotrophic factors NT-4 and NGF- $\beta$ in schizophrenia at two different concentrations of IL-6; * statistically significant differences between the groups $(p<0.05)$

NGF- $\beta$ correlated with the concentration of the proinflammatory cytokine IL-6: upon a significant increase in IL-6, the levels of these neurotrophins also increased (Figure 4). Further studies are needed to find out what immunological and inflammatory profile and/or clinical phenotype is associated with the changes in BDNF and other neurotrophins in patients with schizophrenia.

Prior to this study, we found no reports on multifactorial assessment of immunity mediators, systemic inflammation, and neurotrophic factors in patients with schizophrenia [1]. The authors of study [13] observed an increase in the serum level of neutrophil activation markers (leukocyte elastase, a1-proteinase inhibitor) in schizophrenia; the increase correlated with the severity of the clinical course. Notably, in patients with nonpsychotic mental disorders, similar changes in 
the neutrophil activation markers were found in $50 \%$ of patients only [14]. In a study by Zabotina et al. [15], an increase in the levels of cytokines IL- 6 , IL-1 $\beta$, and TGF- $\beta$ in patients with schizophrenia was detected during the first psychotic episode. No comprehensive studies on the neurotrophic BDNF, NGF- $\beta$, NT-4 in schizophrenia have been published in recent years.

The immunological changes described in the present article were observed regardless of the duration or type of antipsychotic therapy; yet the changes correlated with the phase and dynamics of schizophrenia, thus indicating an association of the immune abnormalities with the pathogenetic mechanisms of the disease.

Personally-significant stimuli and the activity of neural networks in the brain. An expert analysis of physiological parameters produced by the MRIcompatible polygraph for each subject revealed either emotional or neutral reaction to the presented stimuli. Figure 5 shows statistical maps of the brain neural activity obtained with the help of this expert analysis/ or in the absence of this analysis (i.e. considering or not considering the MRI data). The Table shows the anatomical connections of the brain activities.

The present data show that the information coming from the polygraph introduces corrections into the role of neural networks involved in the perception of neutral and personally-significant stimuli. For example, the perception of personally-significant stimuli (taking into account the polygraphy data) is characterized by an activation of neural networks in the associative zones of the left cuneus and precuneus as well as in the ventrolateral prefrontal cortex of both hemispheres, where the centers of working memory and consciousness are located. Perceiving the personally-significant stimuli (unlike the neutral ones) is characterized by an activation of neural networks in area of the left superior frontal gyrus that is associated with human self-consciousness, and also in areas of the right and left superior medial gyrus associated with the executive functions of the brain (the degree of stimulus emotionality was taken into account). Nevertheless, it is worth noting that even without using the polygraph, the personally-significant stimuli (unlike the neutral ones) were found to activate the limbic system areas: I. hippocampus, r. precuneus, r. putamen, as well as extensive zones in the frontal areas. Thus, using the MRI-compatible polygraph allows one to create a group of $\mathrm{fMRI}$ signals for statistical analysis that is based on an objective assessment of physiological reactions to the presented stimuli. In addition, the presence or absence of physiological reactions to personally-significant stimuli can be seen as a measure of damage to the human

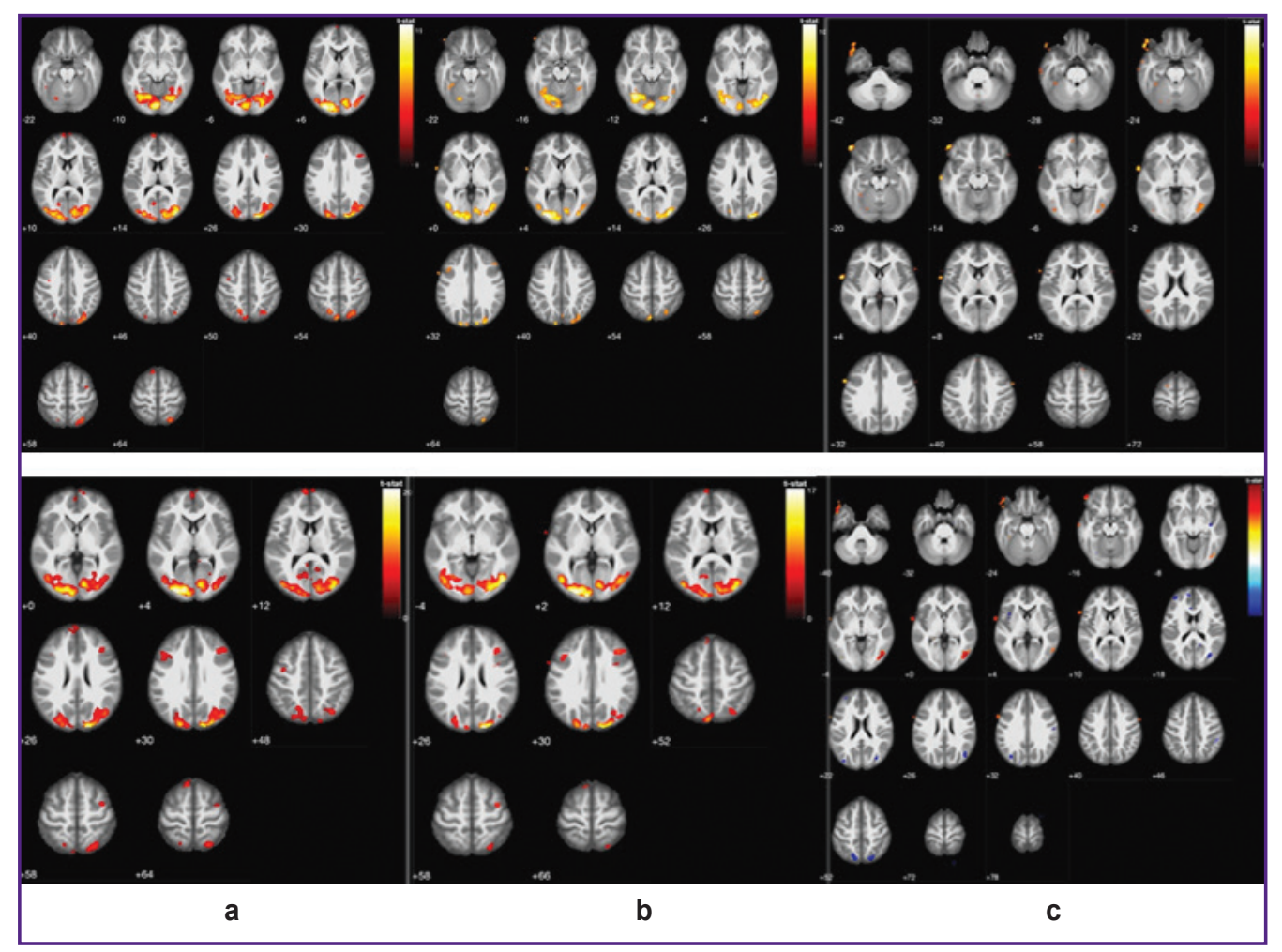

Figure 5. Individual statistical maps placed on a template T1-weighted image

The paradigm with visual stimuli, the comparison considering the analysis of physiological indicators (top) and within the experimental paradigm (bottom): (a) neutral stimuli vs rest; (b) personally significant stimuli vs rest; (c) neutral vs personally significant stimuli; $p<0.05$ corrected for multiple comparisons 


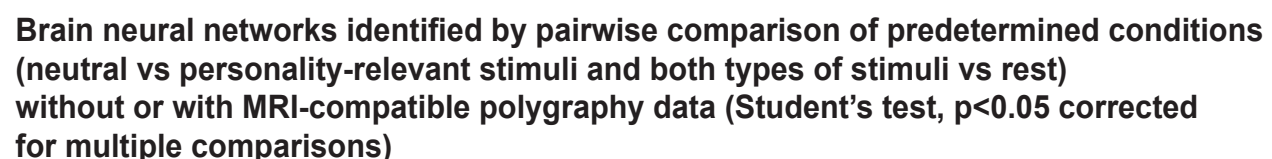

\begin{tabular}{|c|c|c|c|}
\hline \multirow[b]{2}{*}{ Data usage } & \multicolumn{3}{|c|}{ Comparisons } \\
\hline & Neutral stimuli vs rest & $\begin{array}{l}\text { Personality-relevant } \\
\text { stimuli vs rest }\end{array}$ & $\begin{array}{c}\text { Personality-relevant stimuli vs } \\
\text { neutral stimuli }\end{array}$ \\
\hline $\begin{array}{l}\text { Considering } \\
\text { polygraphy data }\end{array}$ & $\begin{array}{l}\text { L. middle occipital gyrus } \\
\text { R. superior occipital gyrus } \\
\text { L. precuneus } \\
\text { L. superior parietal lobule } \\
\text { L. superior frontal gyrus } \\
\text { L. calcarine gyrus } \\
\text { L. superior medial gyrus } \\
\text { L. IFG (p. triangularis) } \\
\text { R. middle frontal gyrus } \\
\text { L. cerebelum (VI) } \\
\text { L. precentral gyrus } \\
\text { L. superior occipital gyrus } \\
\text { R. fusiform gyrus }\end{array}$ & $\begin{array}{l}\text { L. inferior occipital gyrus } \\
\text { L. fusiform gyrus } \\
\text { L. cerebelum (crus 1) } \\
\text { R. middle occipital gyrus } \\
\text { R. superior occipital gyrus } \\
\text { R. inferior temporal gyrus } \\
\text { L. precuneus } \\
\text { L. cuneus } \\
\text { L. middle occipital gyrus } \\
\text { R. superior parietal lobule } \\
\text { R. fusiform gyrus } \\
\text { L. cerebelum (VI) } \\
\text { R. middle frontal gyrus } \\
\text { L. IFG (p. triangularis) } \\
\text { R. IFG (p. triangularis) }\end{array}$ & $\begin{array}{l}\text { R. inferior temporal gyrus } \\
\text { L. inferior occipital gyrus } \\
\text { R. precentral gyrus } \\
\text { L. mid orbital gyrus } \\
\text { L. superior frontal gyrus } \\
\text { L. middle temporal gyrus } \\
\text { L. cerebelum (VI) } \\
\text { L. superior medial gyrus } \\
\text { R. IFG (p. opercularis) } \\
\text { L. cerebelum (crus 1) } \\
\text { L. superior medial gyrus } \\
\text { L. inferior temporal gyrus } \\
\text { Cerebellar vermis }\end{array}$ \\
\hline $\begin{array}{l}\text { Not considering } \\
\text { polygraphy data }\end{array}$ & $\begin{array}{l}\text { L. superior occipital gyrus } \\
\text { R. superior occipital gyrus } \\
\text { R. middle occipital gyrus } \\
\text { L. superior frontal gyrus } \\
\text { R. middle frontal gyrus } \\
\text { L. superior medial gyrus } \\
\text { R. superior medial gyrus } \\
\text { Precentral gyrus } \\
\text { L. middle frontal gyrus } \\
\text { L. precuneus } \\
\text { R. calcarine } \\
\text { R. precuneus }\end{array}$ & $\begin{array}{l}\text { L. calcarine } \\
\text { R. superior occipital gyrus } \\
\text { R. inferior temporal gyrus } \\
\text { R. middle frontal gyrus } \\
\text { L. superior medial gyrus } \\
\text { L. inferior frontal gyrus } \\
\text { L. superior occipital gyrus } \\
\text { R. inferior frontal gyrus }\end{array}$ & $\begin{array}{l}\text { R. IFG (p. triangularis) } \\
\text { L. hippocampus } \\
\text { L. IFG (p. triangularis) } \\
\text { L. fusiform gyrus } \\
\text { R. middle occipital gyrus } \\
\text { L. middle frontal gyrus } \\
\text { R. putamen } \\
\text { L. superior occipital gyrus } \\
\text { R. superior occipital gyrus } \\
\text { L. middle occipital gyrus } \\
\text { R. postcentral } \\
\text { L. insula } \\
\text { L. superior frontal gyrus } \\
\text { R. precuneus } \\
\text { L. superior medial gyrus } \\
\text { R. cerebelum (VI) } \\
\text { R. supramarginal gyrus } \\
\text { L. superior occipital gyrus } \\
\text { R. inferior frontal gyrus } \\
\text { R. calcarine }\end{array}$ \\
\hline
\end{tabular}

limbic system. The proposed method of MRI-compatible polygraphy allows for assessing the activation of neural networks participating in the processes of perception of personally-significant (emotional) information.

\section{Conclusion}

Prior to this study, there were no reports on a multifactorial assessment of neurophysiological indicators and immunity mediators in combination with parameters of systemic inflammation and neurotrophic factors. The first stage of this project allowed us to obtain previously unknown data on neurophysiological characteristics, the cytokine profile and major changes in the immune status of patients with schizophrenia.

The primary results obtained with MRI-compatible polygraphy regarding the visualization of the connectome architecture in a patient perceiving personally-significant stimuli lay the ground for objectively assessing the perception of personally-significant information by selecting a group of $\mathrm{fMRI}$ signals suitable for statistical analysis. At the next stage of the project, we plan to perform a comprehensive analysis of neurophysiological and immunological data at the individual and group levels.

Study funding. This project "Immunogenetic strategies for the diagnosis and treatment of schizophrenia" was supported by the National Research Center "Kurchatov 
Institute" (order No.1604 of July 5, 2018), with partial support from the Russian Foundation for Basic Research: grant 17-29-02518ofi_m "Neuroimmunological status and cognitively effective brain architectures in normal subjects and patients with schizophrenia" and grant 18-29$23020 \mathrm{mk}$ "Study of the functional architecture of human brain neural networks in rest as the basic model of lowenergy information processes of consciousness".

Conflicts of interest. The authors have no conflicts of interest to disclose.

\section{References}

1. Malashenkova I.K., Krynskiy S.A., Ogurtsov D.P., Mamoshina M.V., Zakharova N.V., Ushakov V.L., Velichkovsky B.M., Didkovsky N.A. A role of the immune system in the pathogenesis of schizophrenia. Zhurnal nevrologii $i$ psihiatrii imeni S.S. Korsakova 2018; 118(12): 72-80.

2. Howes O.D., McCutcheon R. Inflammation and the neural diathesis-stress hypothesis of schizophrenia: a reconceptualization. Transl Psychiatry 2017; 7(2): e1024e1024, https://doi.org/10.1038/tp.2016.278.

3. Jacob A., Cohen S., Alavi A. Abnormal brain circuitry and neurophysiology demonstrated by molecular imaging modalities in schizophrenia. J Alzheimers Dis Parkinsonism 2013; 3: 114, https://doi.org/10.4172/2161-0460.1000114.

4. Nenadic I., Smesny S., Schlösser R.G.M., Sauer H., Gaser C. Auditory hallucinations and brain structure in schizophrenia: voxel-based morphometric study. $\mathrm{Br} J$ Psychiatry 2010; 196(5): 412-413, https://doi.org/10.1192/bjp. bp.109.070441.

5. Modinos G., Costafreda S.G., van Tol M.-J., McGuire P.K., Aleman A., Allen P. Neuroanatomy of auditory verbal hallucinations in schizophrenia: a quantitative metaanalysis of voxel-based morphometry studies. Cortex 2013 49(4): 1046-1055, https://doi.org/10.1016/j.cortex.2012.01.009.

6. Allen P., Larøi F., McGuire P.K., Aleman A. The hallucinating brain: a review of structural and functional neuroimaging studies of hallucinations. Neurosci Biobehav Rev 2008; 32(1): 175-191, https://doi.org/10.1016/j. neubiorev.2007.07.012.

7. Downar J., Crawley A.P., Mikulis D.J., Davis K.D.
A multimodal cortical network for the detection of changes in the sensory environment. Neurosci Biobehav Rev 2000; 3(3): 277-283, https://doi.org/10.1038/72991.

8. Bechter K. Updating the mild encephalitis hypothesis of schizophrenia. Prog Neuropsychopharmacol Biol Psychiatry 2013; 42: 71-91, https://doi.org/10.1016/j.pnpbp.2012.06.019.

9. Kinney D.K., Hintz K., Shearer E.M., Barch D.H., Riffin C., Whitley K., Butler R. A unifying hypothesis of schizophrenia: abnormal immune system development may help explain roles of prenatal hazards, post-pubertal onset, stress, genes, climate, infections, and brain dysfunction. Med Hypotheses 2010; 74(3): 555-563, https://doi.org/10.1016/j. mehy.2009.09.040

10. Müller N., Weidinger E., Leitner B., Schwarz M.J. The role of inflammation in schizophrenia. Front Neurosci 2015; 9 : 372, https://doi.org/10.3389/fnins.2015.00372.

11. Vita A., Minelli A., Barlati S., Deste G., Giacopuzzi E., Valsecchi P., Turrina C., Gennarelli M. Treatment-resistant schizophrenia: genetic and neuroimaging correlates. Front Pharmacol 2019; 10: 402, https://doi.org/10.3389/ fphar.2019.00402.

12. Ushakov V.L., Malakhov D.G., Orlov V.A., Kartashov S.I., Kholodny Y.I. Research of neurocognitive mechanisms of revealing of the information concealing by the person. Advances in Intelligent Systems and Computing 2018; 310-315, https://doi.org/10.1007/978-3-319-99316-4_41.

13. Zozulya S.A., Oleichik I.V., Androsova L.V., Otman I.N., Sarmanova Z.V., Stolyarov S.A., Bizyaeva A.S., Yunilainen O.A., Klyushnik T.P. Monitoring trend of endogenous psychoses by immunological parameters. Psikhicheskoe zdorov'e 2017; 15(1): 11-18.

14. Klyushnik T.P., Androsova L.V., Zozulya S.A., Otman I.N., Nikitina V.B., Vetlugina T.P. Comparative analysis of inflammatory markers in endogenous and non-psychotic mental disorders. Sibirskiy vestnik psikhiatrii i narkologii 2018; 2(99): 64-69.

15. Zabotina A.M., Belinskaya M.A., Zhuravlev A.S., Nasyrova R.F., Sosin D.N., Ershov E.E., Taraskina A.E., Krupitsky E.M. Serotonin 2A receptor (HTR2A) gene polymorphisms RS6311 and RS6313 modulate MRNA and protein expression in peripheral blood leukocytes during antipsychotic administration. Tsitologiya 2018; 60(5): 381-389. 